\title{
The Design of Long Distance RFID Reader
}

\author{
Weike Wang ${ }^{1, \text { a }}$, Xinquan Ye ${ }^{2, \mathrm{~b}}$, Meiqing $\mathrm{Li}^{1, \mathrm{c}}$ and Tao $\mathrm{He}^{1, \mathrm{~d} \text {, * }}$ \\ ${ }^{1}$ School of OuJiang, Wenzhou University, WenZhou 325000, China \\ ${ }^{2}$ School of Mechanical and Electrical Engineering, Wenzhou University, WenZhou 325000, China \\ aweikewang113@163.com, b380761381@qq.com, 'andyhe1010@163.com
}

Keywords: Radio Frequency Identification, tag, reader, DSP.

\begin{abstract}
The paper presents a design of HF Reader using a common representative chip for TAGIT tag and CODEl tag. The design of hardware include RF Read/Write module, MCU circuit, serial communicate on circuit, RF power amplifier (PA), signal processing circuit and antenna. In the software design, including the program of read and write operation, anti-collision program, serial communication program between the PC and the Reader, etc. At present, sample machine has been accomplished which could read/write the EPC tag and identify multiple EPC tags.
\end{abstract}

\section{Introduction}

The tags of RFID system in HF band, which receive little energy, are inability to transmit radio waves back to the Reader again. The tag's response signals are weak, which affects the R/W distance of the tag reader. In order to solve this problem, the paper advanced that analog boards and digital boards are developed separately, which improved the reading distance greatly. The reader operating frequency is $13.56 \mathrm{MHz}$ in line with ISO-15693 protocol. The hardware circuit uses TMS320F2812 as the main chip which produced by $\mathrm{Ti}$, aiming at all passive tags complying with ISO-15693 protocol. A good software was design to achieve multi-card identification anti-collision algorithm.

\section{Hardware design of tag reader}

The paper proposed ideas that the analog and digital portions are designed separately, and the hardware design is shown in Figure1. Pulse position code (PPM) Signal is generated by the DSP chip, which then modulates the carrier frequency of $13.56 \mathrm{MHz}$. The power of modulated signal is weak. It is needed to amplify the power first, then filter and tune to supply the antenna, so that to improve the operation distance of the card. The system output power control can be achieved through DSP, the minimum power is $0.25 \mathrm{~W}$. The antenna coil is in the form of impedance while working at $13.56 \mathrm{MHz}$ frequency. In order to match a power of $50 \Omega$ system, it need to convert impedance into resistance through the impedance matching circuit, then connect to end of the reader using the 50 grade coaxial cable. In the receive path, the tag responses signals. Firstly, Sideband is removed by band-pass filter, then amplified and sent into a demodulator, and finally sent the demodulated signal to the DSP chip A/D sampling decision and decoding and verification, which completing the whole process of the received signal. The reader is not only completing simple tag identification, but also forming a large data network via RS232 interface communicating with PC and thus achieving the tags management and operation. The design of the reader is divided into the hardware design and Digital part. The analog portion is the RF modules. The digital parts can be divided mainly Control module, power management modules and external interface module.

Transmission module. The function of transmission module:(1)the $13.56 \mathrm{MHz}$ crystal oscillator produces carrier wave, DSP would send the information and modulation amplitude to the IO of 74HC125 chip, completing ASK modulation (2) NPN RF power transistors achieve power amplification;(3) the carrier frequency will be transmitted out by the antenna through the match circuit receiver module. The function of receiver module:(1) design a narrow-band filter to remove the side of waves, and filter $13.56 \mathrm{MHz}$ frequency components, (2) two stage amplifier circuit (3) The 
sideband signal is to mixed with the local $13.56 \mathrm{MHz}$ carrier waves to modulate onto the $423 \mathrm{kHz}$ single sub-carrier to get the Intermediate frequency signal (4) to amplify IF signal and then envelope detect the original signal (5)to be shap and enlarge byLM385 (4)the demodulated analog signal would be sent to the DSP to sample.

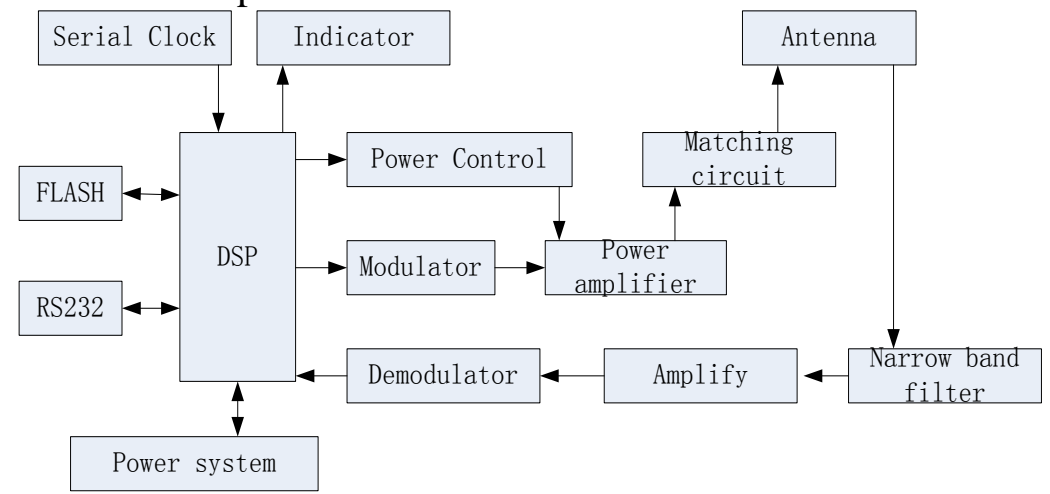

Figure 1 Block diagram of RFID reader hardware

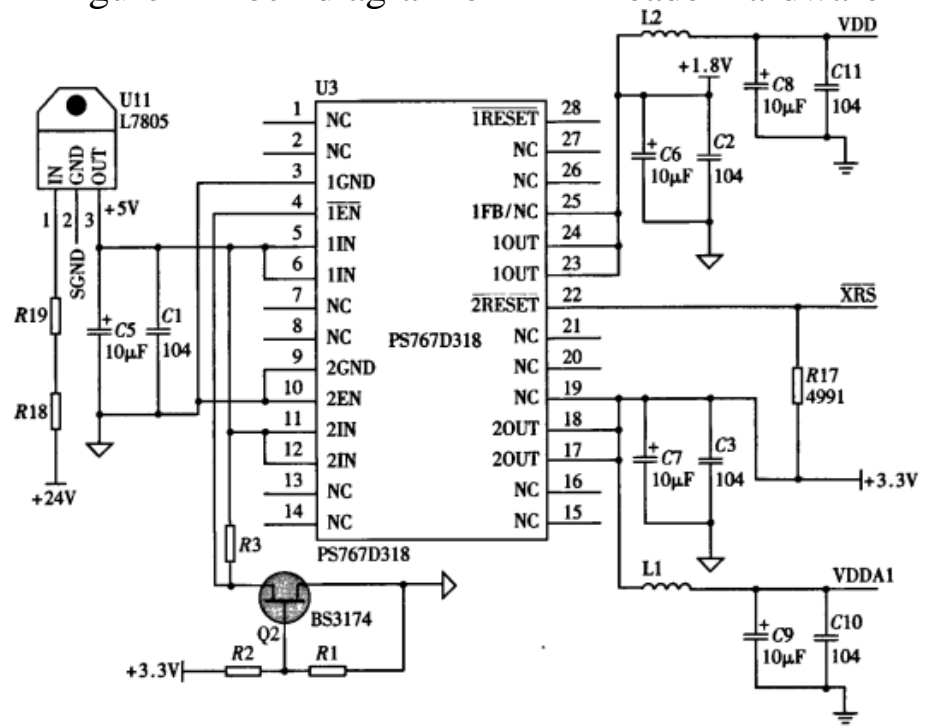

Fig .2 Hardware diagram of RFID Reader

Control module. The system used a DSP chip TMS320F2812 as signal acquisition processing core. In this design, DSP chip is supplied with voltage of $3.3 \mathrm{~V}$ and $1.8 \mathrm{~V}$. The $27.12 \mathrm{MHz}$ crystal oscillator is applied as an external clock circuit, and doubled 5 times through the on-chip phase-locked loop, clock frequency is up to $135.6 \mathrm{MH}$. The function of the master DSP: It is first to collect signal sent by analog board through the 12-bit ADC inside, and the collected data is stored temporarily in the internal storage, then to be the realization of the noise processing Manchester decoding and seizure collision test etc. With the help of software design, and then to judgment and execute specific reading and writing commands and communicate with the host machine through RS232 serial interface.

Power Management Module. The main components of this module are L7805 and PS767D318, the original circuit processing is shown in Figure 2, in which the $24 \mathrm{~V}$ input voltage is adjusted to $5 \mathrm{~V}$ through three terminal regulators L7805. Since the DSP supply voltage is $3.3 \mathrm{~V}$ and $1.8 \mathrm{~V}$, it needs an external dual-output low dropout voltage converter PS767D318 to achieve second grade level conversion.

External Interface Module. The main components of this module is MAX232 chip, which is a bi-directional transmitting and receiving interface lining with EIA RS-232 standard and the V28 specification, realizing the connection with PC through a standard nine-pin serial port.

\section{Reader software design}


Overall design process. It needs a PC monitor when the reader is applied to open access control systems, both of them work in master-slave mode. After completion of power-on reset and initialization, the system will enter into the waiting state. The control module is wake-up by interrupt, then receives and processes the corresponding program, after receiving the instruction sent by the PC. After that, it will return information to PC Machine and enter a waiting state again, the overall process is shown in Figure 3.The reader will begin to perform reception when the interrupts arouse it to send commands and detect the tags entering. The receiving process is shown in Figure 4. After sending the command, the master module will immediately to be ADC sampling for signal from an analog board, and continue to collect noise Sound, followed by the completion of the test data, decoding, verification, anti-collision, and finally the sending information of tag back to the PC, quitting interrupt.

Anti-collision algorithm for multi-card identification. When the reader is in working condition, all tags within its antenna coverage will be activated and get ready to response to the command of the reader, and that will result in tags collision. There are commands such as Inventory, Stay quiet, etc. in IS015693 protocol for multi-card identification. Inventory command is used to check UID (tag identification number) in the antenna coverage, the frame format includes flags, mask length, mask value etc. It needs to customize the length of the slot and add the mask code length and code after the command field when the reader is required to send inventory commands. Assuming mask length, mask value are both 0 , slot length slot is set to 16 , the reader sends inventory command when slot is 0 , the cards with in the working area which number ending in 0 will be the first to response, sending their own UID back in a certain frame format, then when slot is 1 . Readers continue to send EOF, the tags with in the working area which number ending in 1 will to response. And so on, until the reader to send the 15th EOF, there will the end of a complete Inventory command. If there are two tags which's end number are $82 \mathrm{H}$ and $12 \mathrm{H}$ in the work area, they will response to the collision when the slot is 2 . It is necessary to record collision position 2 , set the mask length $=4$ and mask value $=2$, in the next Inventory command, the two tags will response when they their slot is 8 and 1 . So that, the anti-collision problem will be solved. Stay quiet command is used to keep tags which's UIDs are the same with the transmission frame in a quiescent state, no longer respond to any ISO command.

\section{Test}

In the $R \& D$ process, a number of performance measurements of the reader are tested. The main test items and test results are as follows:

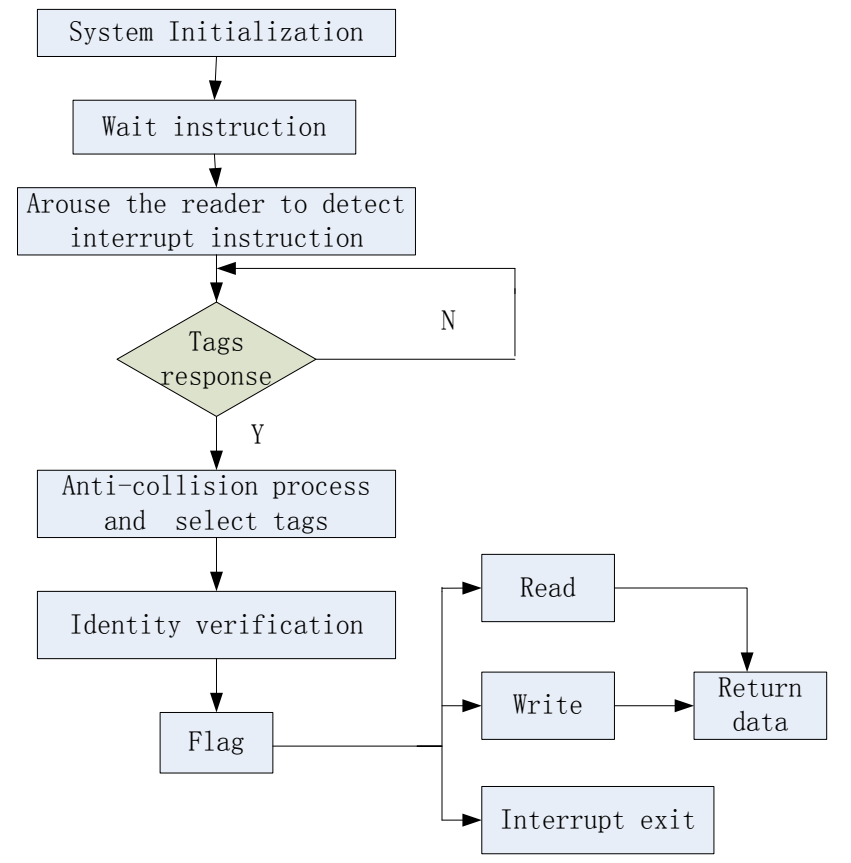

Figure 3 the overall flow chart 


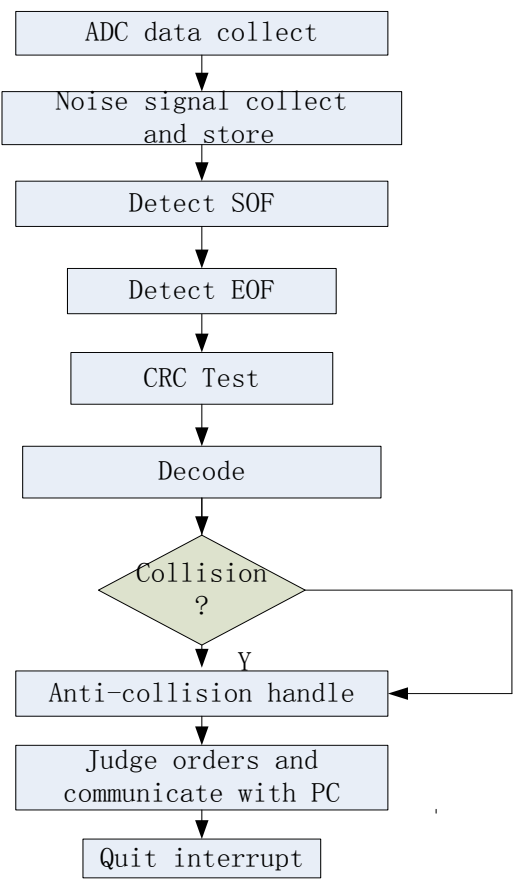

Figure 4 Data reception process

Run Stability: In case of a long time without shutdown, normal operation, no crashes or restart phenomenon, literacy normal working distance $\pm 5 \mathrm{~cm}$ minor fluctuations.

Communication situations with PC: normal.

False leak detection: None.

Anti-collision capability: 40 per second.

Reading distance: about 1.1m single antenna, dual antenna $1.8 \mathrm{~m} \sim 2 \mathrm{~m}$.

Rate question: The fastest speed downlink signal $26.48 \mathrm{~kb} / \mathrm{s}$, the fastest rate uplink $26.69 \mathrm{~kb} / \mathrm{s}$. It is completely full for need of open access control systems. If there are necessary for higher reading distance or speed requirements, such as warehouse management, automatic toll collection systems, the UHF Band and active radio frequency identification system will be the answers.

\section{Acknowledgement}

This paper was supported by Research Projects in the Public Interest of Zhejiang Science and Technology Department No.2012C21050 and No.2012C21086, The Science and Technology Project of Wenzhou Science and Technology Bureau No. KZ1211003 and No.J20120018, The Science and Technology research projects of Zhejiang Education Department No.Y201224800.

\section{References}

[1]EPC Radio-Frequency Identify Protocols Class-l Generation-2 UHF RFID Protocol for Communications at 860MHz.960MHz Version 1.1.0.2005.

[2]RFID world web. Website: http:// www.rfidworld.com.cn.

[3]ISO/IECWD 18000-6REV 1. Information Technology-Radio Frequency Identification (RFID) for ItemManagement-Part6-Parameters for Air Interface Communications at 860-960 IV.2007.

[4]Huizhi Wu, Yunlong Zhu, Haibo Luo. The design of RFID reader based on warehouse management. Application of Electronic Technique, 2006, 32(3):75.

[5]Information on http://www.rfidword.com.cn. 